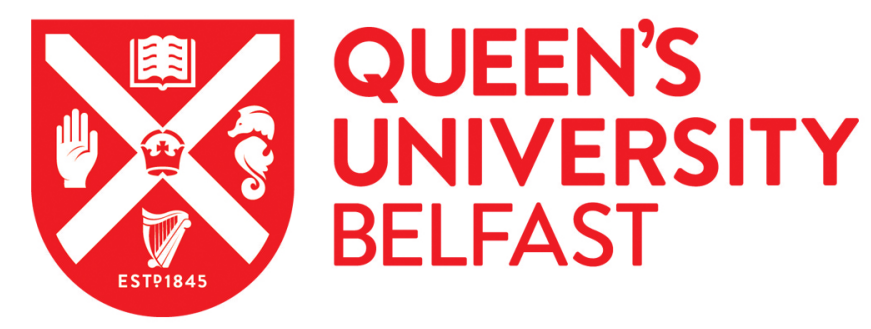

\title{
Weibel-induced filamentation during an ultrafast, laser-driven plasma expansion
}

Quinn, K., Romagnani, L., Ramakrishna, B., Sarri, G., Dieckmann, M., Wilson, P. A., Fuchs, J., Lancia, L., Pipahl, A., Toncian, T., Willi, O., Clarke, R. J., Notley, M., Macchi, A., \& Borghesi, M. (2012). Weibel-induced filamentation during an ultrafast, laser-driven plasma expansion. Physical Review Letters, 108(13), [135001]. https://doi.org/10.1103/PhysRevLett.108.135001

Published in:

Physical Review Letters

Document Version:

Publisher's PDF, also known as Version of record

Queen's University Belfast - Research Portal:

Link to publication record in Queen's University Belfast Research Portal

\section{General rights}

Copyright for the publications made accessible via the Queen's University Belfast Research Portal is retained by the author(s) and / or other copyright owners and it is a condition of accessing these publications that users recognise and abide by the legal requirements associated with these rights.

Take down policy

The Research Portal is Queen's institutional repository that provides access to Queen's research output. Every effort has been made to ensure that content in the Research Portal does not infringe any person's rights, or applicable UK laws. If you discover content in the Research Portal that you believe breaches copyright or violates any law, please contact openaccess@qub.ac.uk. 


\title{
Weibel-Induced Filamentation during an Ultrafast Laser-Driven Plasma Expansion
}

\author{
K. Quinn, ${ }^{1}$ L. Romagnani, ${ }^{2, *}$ B. Ramakrishna, ${ }^{1}$ G. Sarri, ${ }^{1}$ M. E. Dieckmann, ${ }^{1}$ P. A. Wilson, ${ }^{1}$ J. Fuchs, ${ }^{2}$ L. Lancia, ${ }^{2}$ \\ A. Pipahl, ${ }^{3}$ T. Toncian, ${ }^{3}$ O. Willi, ${ }^{3}$ R. J. Clarke, ${ }^{4}$ M. Notley, ${ }^{4}$ A. Macchi, ${ }^{5,6}$ and M. Borghesi ${ }^{1}$ \\ ${ }^{1}$ Centre for Plasma Physics, School of Mathematics and Physics, The Queen's University of Belfast, Belfast BT7 1NN, United Kingdom \\ ${ }^{2}$ LULI, École Polytechnique, CNRS, CEA, UPMC, 91128 Palaiseau, France \\ ${ }^{3}$ Institut für Laser- und Plasmaphysik, Heinrich-Heine-Universität, D-40225 Düsseldorf, Germany \\ ${ }^{4}$ Central Laser Facility, Rutherford Appleton Laboratory, Chilton, Oxfordshire OX11 OQX, United Kingdom \\ ${ }^{5}$ Istituto Nazionale di Ottica, CNR, Pisa, Italy \\ ${ }^{6}$ Dipartimento di Fisica "E. Fermi," Università di Pisa, Pisa, Italy
}

(Received 15 June 2011; published 26 March 2012)

\begin{abstract}
The development of current instabilities behind the front of a cylindrically expanding plasma has been investigated experimentally via proton probing techniques. A multitude of tubelike filamentary structures is observed to form behind the front of a plasma created by irradiating solid-density wire targets with a high-intensity $\left(I \sim 10^{19} \mathrm{~W} / \mathrm{cm}^{2}\right)$, picosecond-duration laser pulse. These filaments exhibit a remarkable degree of stability, persisting for several tens of picoseconds, and appear to be magnetized over a filament length corresponding to several filament radii. Particle-in-cell simulations indicate that their formation can be attributed to a Weibel instability driven by a thermal anisotropy of the electron population. We suggest that these results may have implications in astrophysical scenarios, particularly concerning the problem of the generation of strong, spatially extended and sustained magnetic fields in astrophysical jets.
\end{abstract}

DOI: 10.1103/PhysRevLett.108.135001

PACS numbers: 52.35.Qz, 52.38.Fz, 52.70.Nc

The study of plasma instabilities that may trigger the growth of magnetic fields is important for a deeper understanding of a range of astrophysical phenomena, such as the energetic electromagnetic emissions of gamma ray bursts (GRBs). A GRB is set off by the ultrarelativistic jet that is ejected by a supermassive imploding star. A key astrophysics problem is how the strong magnetic fields in GRBs are generated, which are needed to explain their emissions by electron synchrotron radiation. The filamentation instability of relativistic electron beams is frequently invoked [1], but it remains unclear how highly unstable electron beams could magnetize a GRB jet on astrophysical spatial and temporal scales [2].

Another instability that can generate magnetic fields is the Weibel instability. It concerns in its original form [3] the transverse perturbations that may develop in the presence of an electron component with a bi-Maxwellian electron velocity distribution. Weibel demonstrated that the direction along which the electrons are cooler is parallel to the wave vectors of the aperiodically growing waves. We refer here to this instability as the thermal anisotropydriven Weibel instability (TAWI). TAWI has been studied analytically and numerically [4]. It has not yet received much attention in an astrophysical context, because it is unclear how and if a thermal anisotropy could develop on a large enough scale.

The expansion of a planar plasma front into a vacuum has recently been examined with particle-in-cell (PIC) simulations [5]. The density gradient of the rarefaction wave together with the high electron mobility results in an ambipolar electrostatic field that is driven by the thermal pressure gradient. It reduces the electron momentum along the density gradient and leaves unchanged the other components. The resulting thermal anisotropy in Ref. [5] could trigger the TAWI.

GRB jets are turbulent, and we may expect plasma density variations within the entire jet. The energetic electrons yield high thermal pressure gradients and thus strong ambipolar fields. Therefore, magnetic fields generated via the TAWI can develop over large spatial scales that may be even comparable with the full extent of the plasma jet. What remains unclear is how robust the TAWI is against deviations from a planar slab geometry, which is unlikely to be representative for the jet plasma. It is therefore of interest to study the temporal evolution of the TAWI in nonplanar geometries, with particular attention to the spatial extension, stability, and strength of the magnetic fields.

In this Letter, we present the results of experimental investigations and numerical simulations on the instability of electron currents forming behind the front of a cylindrical expanding plasma. In the experiment, the cylindrical plasma was created by focusing an intense, short duration laser pulse onto solid wire targets. The experimental observations were carried out by employing transverse proton probing, and the electromagnetic fields related to filamented electron currents could be spatially and temporally resolved. Remarkably, it was found that the filaments are frozen into the expanding plasma, exhibiting stability over a time scale (several tens of picoseconds) that largely exceeds the laser irradiation period (less than a picosecond). Comparison of the experimental data with particletracing simulations suggests that a multi-Tesla magnetic 
field is generated over a filament length of several times the filament radii. The filamentation is attributed to the action of a TAWI induced by the loss of radial (in the following, we will refer to the direction normal to the wire surface as the radial direction) momentum of the electrons to the accelerating ions. A PIC simulation verifies that the TAWI can drive magnetic fields with amplitude, growth time, and topology compatible with the experimental observation.

The experiment was carried out by employing the petawatt arm of the Vulcan laser system at the Rutherford Appleton laboratory. In our experiment, the main Vulcan beam, operating in the chirped pulse amplification (CPA) mode, was $1.053 \mu \mathrm{m}$ in wavelength, $\sim 250-350 \mathrm{~J}$ in energy, and $\sim 700$ fs in pulse duration. A first laser beam $\left(\mathrm{CPA}_{1}\right)$ was obtained by extracting $\sim 6 \%$ of the main petawatt beam energy with a $45^{\circ}$ pickup mirror and was focused on wire targets of different diameter and materials (50, 100, and $125 \mu \mathrm{m}$ diameter $\mathrm{Al}$ and $100 \mu \mathrm{m}$ diameter $\mathrm{Au}$ and $\mathrm{CH}$ ) at intensities $\sim 3 \times 10^{19} \mathrm{~W} \mathrm{~cm}^{-2}$. The plasma expanding from the wire targets was probed transversally by employing a diverging, broadband proton beam in the proton projection imaging arrangement (cf. [6] for details on this technique). The proton beam was accelerated in the interaction of the focused remaining part $\left(\mathrm{CPA}_{2}\right)$ of the petawatt beam with $20 \mu \mathrm{m}$ thick gold foils. Protons were detected by employing a multilayer stack of radiochromic films (RCFs) [7]. The distance between the gold foil and the wire target was $4.5 \mathrm{~mm}$, and the distance between the wire target and the RCF stack was $6 \mathrm{~cm}$, leading to a projection magnification of $\sim 14$.

A representative sample of the experimental RCF data obtained is displayed in Fig. 1. The probing times associated to each image $\left(t_{r}\right)$ are expressed relative to the incidence of $\mathrm{CPA}_{1}$ onto the wire. Because of the escape of a fraction of the laser-accelerated electrons into vacuum, the
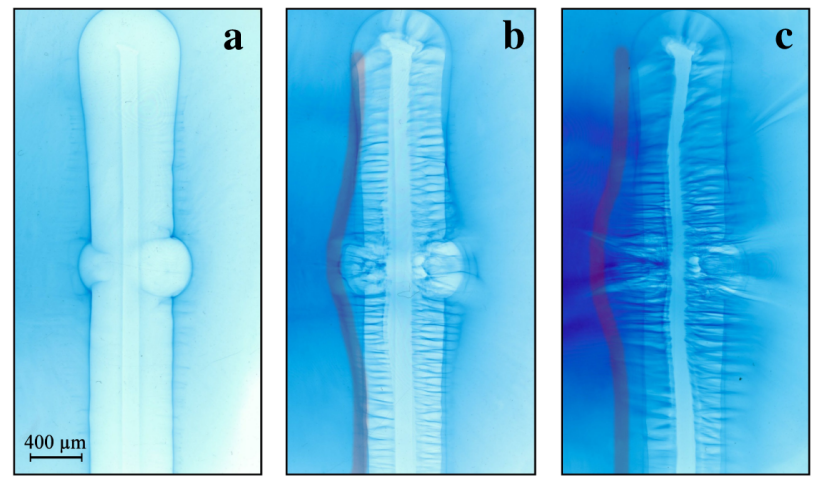

FIG. 1 (color online). Selection of RCF images describing the $\mathrm{CPA}_{1}$-wire interaction with $t_{r}$ values of (a) $\sim 10$, (b) $\sim 30$, and (c) $\sim 50 \mathrm{ps}$. The laser is incident from the right. The faint red lines of images (b) and (c) indicate the approximate positions of the $n_{p}$ pileups caused by the deflection of probe protons by the radially expanding proton front [10]. target develops a positive charge during the interaction. Furthermore, the electron currents associated with the motion of these electrons are capable of generating multi-Tesla magnetic fields in the target region. Immediately thereafter, the region close to the target will be inaccessible to the protons of the probe beam [8]. As this electromagnetic field decays, protons may once again traverse this inner region. The subsequent highly modulated deflection pattern imposed upon the probe protons is the subject of the remainder of this Letter.

At $t_{r} \sim 10$ ps [Fig. 1(a)], some faint traces of $n_{p}$ fluctuations are visible around the wire [8]. By $t_{r} \sim 30 \mathrm{ps}$ [Fig. 1(b)], we observe the presence of striations in $n_{p}$ oriented approximately perpendicular to the wire axis. These local modulations in the proton density are induced rather uniformly in terms of magnitude and spacing over the entire target and are present at vertical displacements of greater than a millimeter from the position of the $\sim 10 \mu \mathrm{m}$-diameter $\mathrm{CPA}_{1}$ focal spot. The observed striations can be attributed to the presence of a highly ordered series of filamentary structures in the plasma surrounding the wire. The size and number of filaments appear to be constant over the late time observation period. Because of the global target charge-up immediately after the laser irradiation, the onset of the filamentation cannot be resolved in detail. However, the fact that at $t_{r} \sim 10 \mathrm{ps}$ the proton density modulations appear less pronounced than at later times indicates that the instability growth time is perhaps of the order of some picoseconds. The characteristic bell-shaped pattern associated with the target normal sheath acceleration [9] driven plasma expansion can be distinguished on both sides of the wire and along the full visible length of the target (cf. Ref. [10]). The faint lines that have been superimposed over Figs. 1(b) and 1(c) denote the front of this expanding plasma. Noticeably, the filaments are always confined between the target surface and the expanding plasma front.

A magnified region from the bottom-right quadrant of the $t_{r}=28 \mathrm{ps}$ RCF image of Fig. 1(b) is displayed in Fig. 2(a). The remarkable periodicity and collimation of the $n_{p}$ striations is visible on immediate inspection of the figure. The average wavelength of the striation pattern may be readily measured to be $\lambda_{n_{p}}=(45 \pm 20) \mu \mathrm{m}$. Only a small selection of the RCF images obtained were displayed in Fig. 1, yet significant $n_{p}$ modulations persist in additional images associated to relative probing times of 60 and $90 \mathrm{ps}$ (not shown for brevity). Intriguingly, in a separate experiment conducted by using the same laser system, similar structures have been observed via optical shadowgraphy to have persisted for many hundreds of picoseconds [11].

Particle tracing simulations were used to investigate the likely distribution of the electromagnetic fields responsible for the observed proton density modulations in the RCF data. The deflections enacted on the probe protons that give 

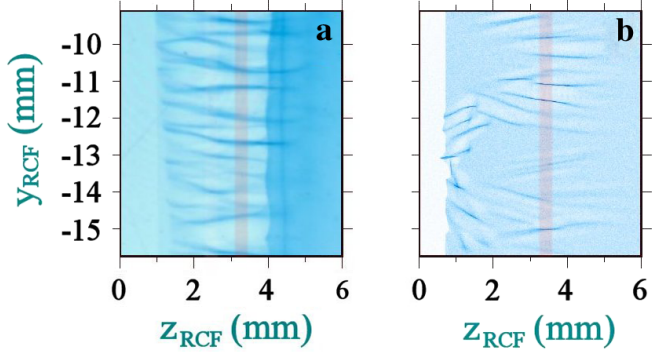

FIG. 2 (color online). (a) Magnified region from the bottomright quadrant of Fig. 1(b). The axis of the wire image runs along the left-hand margin. (b) Matched particle tracing output obtained with $B_{p}=15 \mathrm{~T}, E_{p}=6 \times 10^{8} \mathrm{~V} \mathrm{~m}^{-1}$, and $d=40 \mu \mathrm{m}$.

rise to the observed striations in the proton images are attributed mainly to the action of a magnetic field, while an upper limit for the electric field is found that applies in order to preserve the integrity of the filament projections in the proton image. The fields may be assumed to have been static with respect to the transit time of probe protons through the plasma. The experiment is described in the particle tracer by cylindrical $(r, \theta, y)$ geometry in which the wire axis is oriented along $\hat{\mathbf{y}}$. Several possible spatial field configurations were investigated, but the experimental data were found to have been most closely replicated when the filaments are modeled as a series of magnetic field tubes originating from the wire surface, with a superimposed electric field directed along the tube radii. The tube axes are set along the radial direction $\hat{\mathbf{r}}$ and the filaments distributed randomly in space (see Fig. 3). The radial coordinate and the azimuth angle in the cylindrical coordinate system attached to the filament are, respectively, $\rho$ and $\tilde{\theta}$. The average spacing between the filaments at the wire surface is set to $d$, and the filament radius is in turn set by $\rho_{f}=(d / 2)$. The magnetic field $\mathbf{B}_{\tilde{\theta}}$ encircling each filament and the electric field $\mathbf{E}_{\rho}$ are set to zero both at the filament axis and for $\rho \geq \rho_{f}$, rising sinusoidally with $\rho$ up to the peak values $\left(B_{p}\right.$ and $\left.E_{p}\right)$ in the intermediate region. The variation of $\left|\mathbf{B}_{\tilde{\theta}}\right|$ and $\left|\mathbf{E}_{\rho}\right|$ with increasing distance from the wire surface (i.e., with increasing $r$ ) is controlled via a multiterm sinusoidal function. Particle tracing simulations were conducted across the parameter

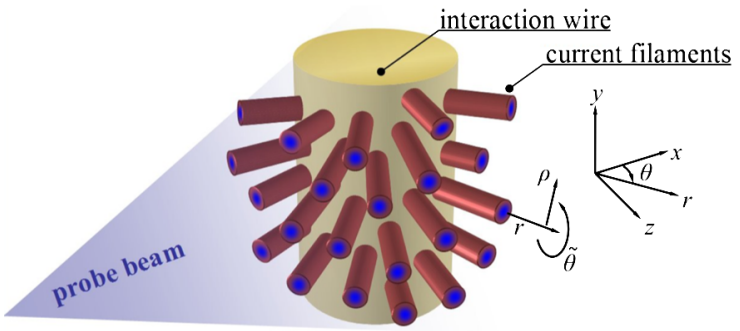

FIG. 3 (color online). Schematic describing the random placement of tubular filaments originating from the wire surface and the coordinate system used in the particle tracing simulations. space of $\left(B_{p}, E_{p}, d\right)$. A closest match between the experimentally observed and simulated $n_{p}$ striations is obtained for $B_{p} \simeq 15 \mathrm{~T}, E_{p} \simeq 6 \times 10^{8} \mathrm{~V} \mathrm{~m}^{-1}$, and $d \simeq 40 \mu \mathrm{m}$ [see Fig. 2(b)], and the upper limit for the electric field amplitude is found to be $\sim 10^{9} \mathrm{~V} \mathrm{~m}^{-1}$. The fields' amplitude can be considered constant at the later observation times within the uncertainties of the field retrieval procedure, as it is found by comparing the data from different RCFs in the film stack with particle tracings for the corresponding proton energies. Note that, even if the filaments are set perpendicularly to the target, in the simulated image (as in the experimental proton image) the projection of some of the filaments appears to be nonorthogonal to the wire projection. This can be explained in terms of the geometrical projection of the three-dimensional (3D) filament distribution onto the proton detector plane.

We then investigated the physical origin and nature of the observed filaments, assuming a collisionless plasma. The radial acceleration of the ions by target normal sheath acceleration and the cylindrical geometry of the expansion front observed in Fig. 1(a) should result in a transfer of radial momentum from the electrons to the ions; the electron's radial thermal energy $T_{r}(r)$ decreases compared to the $T_{\theta}$ and $T_{y}$ in the azimuthal and axial directions. A thermal anisotropy $A(r)=T_{r}(r) / T_{\theta}(r)<1$ results in a TAWI.

A two-dimensional (2D) PIC simulation was carried out to support our interpretation. It resolves the plane orthogonal to the wire axis $y$. Maxwell's equations are solved, together with the relativistic Lorentz force equation [12], to evolve in time the electromagnetic fields and the trajectories of the computational particles. A circle with radius $r_{w}$ corresponds to the wire's cross section that is initially surrounded by a vacuum. The wire is represented by electrons and protons with the correct mass ratio. The plasma is initially charge and current neutral, and no initial or external electromagnetic fields, e.g., a laser pulse, were introduced. The plasma frequency $\omega_{p e 0}=$ $5.64 \times 10^{13} \mathrm{~s}^{-1}$ is well below that within a wire with its solid density, but in reality most of the electrons are confined by practically immobile heavier ions and cannot move beyond the wire surface; the wire in Fig. 1 remains intact. The wire radius is $r_{W}=0.29 \mathrm{~mm}$ or 54.5 electron skin depths $c / \omega_{p 0}$. The proton (electron) temperature is $10 \mathrm{eV}$ $(32 \mathrm{keV})$. The electron thermal pressure drives the cloud expansion, and the most energetic protons reach $\sim 0.026 c$ (corresponding to just 0.1 times the electron thermal speed of $0.25 c$ ) during the simulation time $t_{s}=20 \mathrm{ps}$.

The supplementary video [13] shows the time-evolving simulation data, and a snapshot at $t=10 \mathrm{ps}$ is displayed by Fig. 4. The normalized plasma frequency $\omega_{p e}(r)$ is calculated from the averaged electron density $n_{e}(r)=$ $(2 \pi r)^{-1} \int_{\theta} n_{e}(r, \theta) r d \theta$ and displayed in Fig. 4(a). The thermal anisotropy $A(r)=T_{r}(r) / T_{\theta}(r)$ is also shown, together with the normalized magnetic energy density 

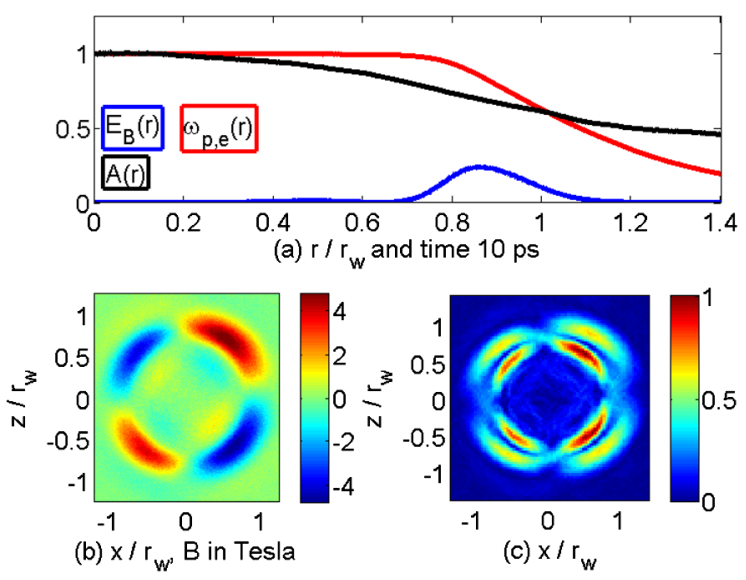

FIG. 4 (color online). Sample of PIC simulation results. (a) Electron plasma frequency $\omega_{p e}$ (in units of the initial electron plasma frequency $\omega_{p 0}$ ), thermal anisotropy $A(r)$, and magnetic energy density $E_{B}(r)$. (b) Magnetic field $B_{y}(x, z)$ and (c) total current density $J$ (in units of the maximum current). The results are plotted at the time $t=10 \mathrm{ps}$.

$E_{B}(r) \propto r^{-1} \int_{\theta} B_{y}^{2}(r, \theta) r d \theta$. Only $B_{y}$ can grow in response to the TAWI in the chosen geometry.

A decrease of $\omega_{p e}$ can be seen for $r>0.7 r_{W}$. The electron density follows the proton density profile of the rarefaction wave. The steady decrease of $A(r)$ for $r>0.2 r_{W}$ can be attributed to the electron's loss of momentum to the electrostatic field of the rarefaction wave. The magnetic energy density peaks in the interval $0.7<r / r_{W}<1.2$, and a weaker maximum is observed at a lower $r$. The $B_{y}(x, z)$ in Fig. 4(b) reveals a structure, which is periodic in the $\theta$ direction and performs one nonsinusoidal oscillation in the radial direction. The magnetic structure emerges from noise at $t=6 \mathrm{ps}$. It is periodic along $\theta$, and it performs initially 3 and later on 2 oscillations along $\theta$. The amplitude grows to $4 \mathrm{~T}$ at $t=10 \mathrm{ps}$ and to a peak value of $11 \mathrm{~T}$ at $t=13 \mathrm{ps}$ [13], which is close to the above estimate of $15 \mathrm{~T}$, while the instability growth time is compatible with the experimental observation. At later times ( $t \gtrsim 15 \mathrm{ps})$ the magnetic field starts decaying and losing its ordered structure. The lifetime of the stationary magnetic structures is somewhat less than the experimentally observed one, presumably due to 2D geometry and because the ratio of hot electrons to expanding ions is much smaller than in the experiment.

In summary, a cohesive picture of the nature and origin of long-lived, spatially extended filamentary structures observed, via proton probing, behind the front of a cylindrically expanding plasma has been presented. It has been demonstrated via the use of particle tracing simulations that the observed filaments may be modeled as a series of magnetized tubes extending radially from the wire target and being randomly distributed in space. The mechanism responsible for the formation of the observed filaments has been identified with a Weibel instability driven by a thermal anisotropy of the electrons in the target region. This model has been supported qualitatively with a 2D PIC simulation, in which a plasma represented the cross section of the wire. A similar anisotropy-driven instability in a plane containing the wire axis [5] will result in a closure of the magnetic fields around the current channels. We suggest that these results may have implications for astrophysical scenarios, where TAWI could produce strong magnetic fields over large spatial and temporal scales in astrophysical plasma jets.

This research was supported by EPSRC Grants No. EP/ E048668/1, No. EP/E035728/1, and No. EP/C003586/1; DFG No. TR18 and No. GK1203; the British Council Alliance Program; QUB Internationalization Funds; DEL/AWE plc CAST; the STFC Direct Access Scheme; Vetenskapsradet (Dnr: 2010-4063); EPSRC/Andor Technology; Leverhulme Trust fellowship (ECF-2011383); and MIUR, Italy through the FIRB project "SULDIS". The support of the staff of the Central Laser Facility at RAL is gratefully acknowledged. L. R. acknowledges support from the ULIMAC grant from the Triangle de la Physique RTRA network.

*lorenzo.romagnani@polytechnique.edu

[1] M. V. Medvedev and A. Loeb, Astrophys. J. 526, 697 (1999).

[2] E. Waxman, Plasma Phys. Controlled Fusion 48, B137 (2006).

[3] E. S. Weibel, Phys. Rev. Lett. 2, 83 (1959).

[4] F. Pegoraro, Phys. Scr. T63, 262 (1996); A. Macchi, A. Antonicci, S. Atzeni, D. Batani, F. Califano, F. Cornolti, J. J. Honrubia, T. V. Lisseikina, F. Pegoraro, and M. Temporal, Nucl. Fusion 43, 362 (2003); V. K. Tripathi and C.S. Liu, Phys. Fluids 25, 629 (1982); R. L. Morse and C.W. Nielson, Phys. Fluids 14, 830 (1971); A. Stockem, M.E. Dieckmann, and R. Schlickeiser, Plasma Phys. Controlled Fusion 51, 075014 (2009); Y. Sentoku, K. Mima, Z. M. Sheng, P. Kaw, K. Nishihara, and K. Nishikawa, Phys. Rev. E 65, 046408 (2002).

[5] C. Thaury, P. Mora, A. Héron, and J. C. Adam, Phys. Rev. E 82, 016408 (2010).

[6] M. Borghesi et al., Appl. Phys. Lett. 82, 1529 (2003); A. J. Mackinnon et al., Rev. Sci. Instrum. 75, 3531 (2004).

[7] M. J. Butson, P. K. N. Yu, T. Cheung, and P. Metcalfe, Mater. Sci. Eng., R 41, 61 (2003).

[8] K. Quinn et al., Phys. Rev. Lett. 102, 194801 (2009).

[9] S. C. Wilks, A. B. Langdon, T.E. Cowan, M. Roth, M. Singh, S. Hatchett, M.H. Key, D. Pennington, A. MacKinnon, and R. A. Snavely, Phys. Plasmas 8, 542 (2001).

[10] L. Romagnani et al., Phys. Rev. Lett. 95, 195001 (2005).

[11] S. Kar (private communication).

[12] J. W. Eastwood, Comput. Phys. Commun. 64, 252 (1991).

[13] See Supplemental Material at http://link.aps.org/ supplemental/10.1103/PhysRevLett.108.135001 for a time animation of the PIC simulation. 